\title{
Protection of trabecular meshwork cells by eyedrops containing high concentration of polyphenols
}

\author{
Sergio C Sacca ${ }^{1}$, Alessandra Pulliero ${ }^{2}$, Sebastiano La Maestra ${ }^{2}$, Marta Geretto ${ }^{2}$, Aldo Profumo ${ }^{1}$, Oralbek Ilderbayev , Camillo Rosano $^{1}$ and $^{3}$ \\ Alberto Izzotti ${ }^{1,2 *}$ \\ ${ }^{1}$ IRCCS Ospedale Policlinico San Martino, Largo Rosanna Benzi 10, 16132 Genoa, Italy \\ ${ }^{2}$ Department of Health Sciences, University of Genoa, Via A. Pastore 1, 16132 Genoa, Italy \\ ${ }^{3}$ Institute of Cell Biology and Biotechnology, L.N. Gumilyov Eurasian National University, Astana, Kazakhstan
}

\begin{abstract}
Background: Glaucoma is caused by pro-apoptotic signals targeting retinal ganglion cells. In the high-pressure glaucoma, the source of these signals is the progressive degeneration of the trabecular meshwork. The first pathogenic event affecting this tissue is oxidative damage. Accordingly, the use of anti-oxidant to counteract glaucoma pathogenic cascade is a rationale strategy to be explored. In this paper we evaluate the ability of eyedrops containing high dose of polyphenols, hyaluronic acid and fatty acids to protect trabecular meshwork from oxidative stress.
\end{abstract}

Methods: Human Trabecular meshwork endothelial cells were exposed in vitro to hydrogen peroxide either in presence or absence of eyedrops containing high dose of polyphenols. Ex-vivo experiments were performed in calf eye to evaluate the ability of eyedrops to cross cornea either alone or complexed with lipid nanoparticles.

The tolerability of eyedrops was tested in vivo in rabbit eye.

Results: Polyphenol enriched eyedrops were highly safe when used alone not altering viability, mitochondrial function and DNA integrity of trabecular meshwork cells. Eyedrops was effective in protecting trabecular meshwork cells from oxidative stress. These characteristics were not modified when eyedrops was carried by lipid nanoparticles.

In calf eye polyphenolic eyedrops were able to cross cornea, an effect counteracted by the complexation with lipid nanoparticles.

In vivo in rabbit eye, eyedrops were well tolerated and persisted for $20 \mathrm{~min}$ on outer corneal surface, a time is increased up to $16 \mathrm{~h}$ by complexation with nanoparticles.

Conclusions: Polyphenolic eyedrops are safe and able to protect trabecular meshwork cells from oxidative stress.

Abbreviations: POAG: Primary Open Angle Glaucoma; TM: Trabecular Meshwork; NP: Lipid Nanoparticles; HDPE: High Dose Polyphenols Extract, SDPE: Standard Dose Polyphenols Extract.

\section{Introduction}

Glaucoma is a multi-factorial disease triggered by apoptotic signals arriving at the head of the optic nerve and resulting in retinal ganglion cells death $[1,2]$. Oxidative stress plays a central role in pathogenesis and progression of primary open angle glaucoma (POAG) [3]. The cause of the imbalance in favor of oxidative damage has been identified in mitochondrial damage affecting trabecular meshwork (TM) cells [4,5]. Furthermore, TM is the most sensitive tissue of the anterior ocular chamber to oxidative stress [6]. POAG is characterized by a remarkable increase of oxidative damage in TM [7], whose consequence is the failure to fulfill its function to regulate aqueous humor outflow and the release of pro-apoptotic signals $[8,9]$.

A tight relationship exists between TM damage and retinal ganglion cells death. Indeed, damaged TM cells release molecular proapoptotic signals reaching retinal ganglion cells through the episcleral pathway [1]. These signals also include extracellular microRNAs [10].

TM damage in glaucomatous patients is directly proportional to intraocular pressure increase and to visual field damage [7]. TM is not a simple passive filter for aqueous humor, conversely it is a complex set of specialized cells actively changing their morphology to expose more or less surface to the aqueous humor thus regulating its outflow from the anterior chamber. Damaged TM cells release proteins into the aqueous humor of glaucomatous patients, as demonstrated by proteomic analyses [4]. The extruded from TM damaged cells are signals to the retina, which consequently suffers because subjected to these pro-apoptotic signals. The retinal ganglion cells as well as cells of the upper neural center cells (i.e., geniculate ganglion and occipital cortex) die because the activation of intrinsic apoptosis resulting from mitochondria damage $[11,12]$. The axon of the optic nerve head, where it folds and passes through the lamina cribrosa, is rich in mitochondria thus being highly sensitive to the activation of intrinsic apoptosis related with mitochondrial damage. Inflammation contributes to ganglion cell damage as demonstrated by the presence of inflammatory cytokines in the aqueous humor of glaucomatous patients $[13,14]$. Therefore new

${ }^{*}$ Correspondence to: Alberto Izzotti, MD, $\mathrm{PhD}$, Department of Health Sciences, University of Genoa, Via A. Pastore, 1, 16132, Genoa, Italy; E-mail: izzotti@unige.it

Key words: polyphenols, glaucoma, trabecular meshwork, oxidative stress, eyedrops Received: February 04, 2019; Accepted: February 13, 2019; Published: February 21,2019 
glaucoma therapies should act both on defense from oxidative stress and inflammation.

Diet and vitamin supplementation have been suggested as having some role on glaucoma. The possible role of long-chain polyunsaturated fatty acids has been explored. A fatty acid imbalance between omega- 3 and omega- 6 is believed to contribute to the pathogenesis of POAG [15] as also suggested by epidemiological studies [16]. However, in clinical trials administering omega-3 supplements did not yield any benefit to POAG patients [17]. Polyphenols have been found to favorably influence inner retinal function in eyes with early to moderately advanced glaucomatous damage. For example, some clinical trials have shown that anthocyanins and Ginkgo biloba extract, rich in polyphenols, may improve the visual function of patients with normal-tension glaucoma $[18,19]$. Short-term supplementation of epigallo- catechin-gallate (EGCG) is neuroprotective in retinal neurons affected by glaucomatous degeneration [20]. Resveratrol has been found to prevent the expression of glaucoma markers induced by chronic oxidative stress and exert anti-apoptotic effects in trabecular meshwork cells [21]. Treatment with resveratrol can prevent trabecular meshwork cell injury caused by oxidative stress in the development of glaucoma [22]. Indeed, red wines strongly inhibit the synthesis of endothelin-1, a vasoactive peptide that plays a crucial role in the pathogenesis of glaucoma [23].

Polyphenols are products of plant metabolism whose main tasks are defense against pathogens and ultraviolet radiation [24]. These molecules are responsible for many food characteristics, such as flavor, color, bitterness and oxidative stability. Since the end of the 20th century, several studies have highlighted that diets rich in polyphenols are recommended for protection against different pathological conditions, such as cancer, diabetes, osteoporosis, cardiovascular and neurodegenerative diseases $[25,26]$. The main property of polyphenols is their ability to act as antioxidant molecules, defending cells against free-radicals that are formed as a consequence of smoking, pollution, rancid foods consumption and physiological metabolism. Moreover, polyphenols are expected to contribute to body anti-inflammatory state reducing the risk of developing chronic diseases.

Regarding polyphenol content in food, this generally depends on environmental factors such as sun exposure, rainfall and soil characteristics among others. The concentration and proportions of different polyphenols are affected by degree of ripeness [27]. Plants contains a lot of polyphenols and nutraceutical molecules such as apigenin, ascorbic-acid, beta-carotene, luteolin, caffeic-acid, alphalinoleic and linoleic acid (omega- 3 and omega- 6 poly-unsaturated fatty acids), oleic acid (mono-unsaturated fatty acid) with a beneficial effect on human health. Coleman et al. [28] found a decreased glaucoma risk in women who consumed fruits and vegetables rich in vitamin $\mathrm{A}$ and carotenes, namely collard greens, kale, carrots, and peaches. Another study by Giaconi et al. [29] also concluded that there is a decreased likelihood of glaucoma among women that reported a greater intake of fruits and vegetables rich in vitamins $\mathrm{A}$ and $\mathrm{C}$, and carotenes.

However, dietary antioxidants are mainly compartmentalized in the liver and blood and barely reach eye tissues also including TM. To face this problem, the adoption of mixture of polyphenols bearing a variety of pharmacodynamic properties and characterized by high bioavailability and long persistence in the eye could be a new promising strategy for glaucoma therapy and prevention.

The present paper aims at evaluating safety and efficacy of a formulation of high-dose polyphenols and fatty acids (hyaluronic acid) in protecting TM from oxidative damage.

\section{Materials and methods}

\section{Eyedrops}

Eyedrops was tested both as extract at high $(0.15 \% \mathrm{w} / \mathrm{v})$ (high dose polyphenols extract, in hyaluronic acid $0,25 \%$ HDPE (iTRAB) or complexed with nanoparticles, HDPE-NP (ERISnp ${ }^{\circ}$ ) versus standard $(0.1 \% \mathrm{w} / \mathrm{v})$ (standard dose polyphenols extract, SDPE) polyphenol concentration.

HDPE derives from a mixture of Perilla extracts in which different polyphenols and fatty acids have been included. The high concentration drug was used as a commercially available (DRAIN drops ${ }^{\bullet}$, Eye Pharma $\mathrm{SpA}$, Genoa, Italy). All solvents and components other than polyphenols and fatty acids were similar in the two mixtures (hyaluronic acid with high molecular weight, excipients).

\section{LC/MS analysis}

Polyphenols contained in HDPE were identified by GC/MS analysis. The dried HDPE powder was dissolved in acetonitrile (final concentration $0.1 \mathrm{mg} / \mathrm{mL}$ ). The sample qualitative composition was determined by high performance liquid chromatography (HPLC)/mass spectrometry (MS) (Agilent series 1200, Agilent Technologies, Palo Alto, CA, USA). Samples ( 5 ul) were into C18 column (Waters, Milford, MS, USA). Mobile phases A was $0.1 \%$ formic acid in water; mobile phase $\mathrm{B}$ was $0.1 \%$ formic acid in acetonitrile. The flow rate was $20 \mathrm{uL}$. The eluent flow was directly sent to the electrospray (ESI) ion source of the 6210 Time of Flight mass spectrometer (Agilent Technology) in order to characterize the HPLC peaks. The parameters applied were: 4300 $\mathrm{V}$; nebulizer pressure: 20 psig; drying gas: $5 \mathrm{~L} / \mathrm{min}$; gas temperature: $300^{\circ} \mathrm{C}$; fragmented voltage: $250 \mathrm{~V}$; skimmer voltage: $80 \mathrm{~V}$; octapole RF: 250 V. Mass Hunter Qualitative Analysis (Agilent Technology) was used to process the full-scan data, previously recorded through the Agilent's Mass Hunter software. Mass spectra, ranging from 100-2000 m/z, were acquired both in reflectron positive and negative ion mode and the presence of selected analytes (fatty acids, catechins, polyphenols, etc.) was evaluated by extracted ion current (EIC) based on their theoretical molecular weight $[\mathrm{M}+\mathrm{H}]+$ or $[\mathrm{M}-\mathrm{H}]-$. During the acquisition of mass spectra the internal mass calibration was automatically performed by the instrument through the use of a dual nebulizer electrospray source with an automated calibrant delivery system.

\section{In vitro studies}

Human Trabecular meshwork endothelial cell line at 5th replication passage were used (HTM cells, ScienCell Research Laboratories, CA, USA). Cells were grown until semiconfluence (80\% plate coverage) in $10 \% \mathrm{v} / \mathrm{v}$ calf serum, $2 \mathrm{mM} \mathrm{L}$-glutamine, penicillin $(100 \mathrm{IU} / \mathrm{ml})$ at $37^{\circ} \mathrm{C}, \mathrm{CO}_{2} 5 \% \mathrm{v} / \mathrm{v}$. HDPE safety was evaluated by analyzing cell modification induced when the drug was used alone. HDPE efficacy was evaluated as the protection exerted in trabecular meshwork cells against oxidative stress induced by hydrogen peroxide.

The following experimental conditions were set up:

1. Untreated control

2. HDPE $50 \mathrm{ul}$ per well for $30 \mathrm{~min}$

3. Hydrogen peroxide $50 \mathrm{uM}$ for $10 \mathrm{~min}\left(\mathrm{H}_{2} \mathrm{O}_{2}\right)$

4. HDPE pre-treatment $+\mathrm{H}_{2} \mathrm{O}_{2}$ 
5. HDPE co-treatment $+\mathrm{H}_{2} \mathrm{O}_{2}$

\section{6. $\mathrm{H}_{2} \mathrm{O}_{2}+\mathrm{HDPE}$ post-treatment}

After treatments, cells were tested for viability and genotoxic damage (DNA fragmentation).

Cell viability (MTT test): Cells were collected by tripsin/EDTA treatment and transferred onto multiwell plates $\left(7 \times 10^{3}\right.$ cells per well) and viability measured by 3-(4,5-dimethylthiazol-2-yl)-2,5 diphenyl tetrazoliumbromide (Sigma Chemical Company, St. Louis, MO, USA) metabolization test (MTT). MTT is metabolized into mitochondria of living cells into metabolites absorbing light at 570 $\mathrm{nm}$. MTT $(5 \mathrm{mg} / \mathrm{ml}$ in PBS) was added to each well and incubation performed at $37^{\circ} \mathrm{C}$ for $3 \mathrm{~h}$. Absorbance at $570 \mathrm{~nm}$ was analyzed by multi-well photometer (Multiskan FC, Thermo Fisher Scientific, Walthman, Massachusetts, USA). For each experimental condition 5 independent replicate experiments have been performed. Viability was expressed as percentage of absorbance as compared to $100 \%$ observed in controls. Results were analyzed by ANOVA using statistical package (Statview, SAS, NC, USA).

Genotoxic damage (comet test): Comet test evaluates DNA fragmentation by alkaline gel electrophoresis determining the presence of apurinic sites and strand breakage resulting from 8-oxo-dG formation. Cells were suspended in agarose $0.5 \% \mathrm{w} / \mathrm{v}$ and distributed onto microscope slide. Cells were lysed by Triton X $1 \%$ in buffered solution and alkalinized by $\mathrm{NaOH}(0.3 \mathrm{M}, \mathrm{pH} 13)$. Electrophoresis was run for $30 \mathrm{~min}$ at $25 \mathrm{~V}, 0.66 \mathrm{~V} / \mathrm{cm}, 300 \mathrm{~mA}$. Cells were dried by ethanol $100 \%$, and DNA stained by ethidium bromide. Slides were analyzed at 400x by fluorescence microscopy (Leica DMI 4000B) equipped with digital camera (Leica DFC 310FX). 100 nuclei per slide were examined by a CASP software (http://www.casp.sourceforge.net). DNA breakage was quantified as tail moment calculated multiplying tail length for the fraction of total DNA contained in the tail. Differences among experimental groups were evaluated by ANOVA.

\section{Ex vivo study: Evaluation of corneal distribution}

The ability of eye-drops, either un-carried or carried by lipid nanoparticles, to penetrate corneal membrane was tested in calf eye collected from cadaver. Eyedrop was charged onto lipid nanoparticles (diameter $70 \mathrm{~nm}$ ) (NP) by $15 \mathrm{~min}$ incubation at room temperature [30]. These nanoparticles are characterized by (a) cationic groups on surface binding to negatively charged cell membranes; (b) ability of carrying lipophilic drugs in the lipid interface. HDPE was labelled by fluorescing, applied onto ocular anterior surface for $30 \mathrm{~min}$ and corneal distribution evaluated by slit lamp.

The amount of HDPE passing through corneal membrane was quantified by spectrofluorimeter (F3000, Hitachi, Tokyo, Japan) (Ex $500 \mathrm{~nm}$, Em $561 \mathrm{~nm}$ ).

\section{In vivo study}

Fluorescin-labeled HDPE was applied onto the ocular anterior surface of 5 male rabbits and eyedrop persistence determined by blue light irradiation. HDPE was used either alone (iTRAB ${ }^{\circledR}$ ) (left eyes) or complexed with lipid nanoparticles (HDPE-NP (ERISnp ${ }^{\star}$ ) (right eyes). Eyedrop persistence on the ocular anterior surface was evaluated by blue light irradiation at 5,10,20,30,45, 60, $90 \mathrm{~min} ; 2,3,4$ and $16 \mathrm{~h}$ time intervals. The presence of inflammation in conjunctiva was examined by direct observation at the moment of treatment and for 1 week thereafter.
The animal experimental protocol was approved by the Institute of Cell Biology and Biotechnology, L.N. Gumilyov Eurasian National University, Astana, Kazakhstan. Housing and treatments of animals were in accordance with NIH, European (2010/63 UE Directive), and institutional guidelines. The issuance of the NIH Office of Laboratory Animal Welfare (OLAW) with the University of Genoa bears the identification number A5899-01 and is effective until February 28, 2021.

\section{Results \\ LC/MS analysis}

LC/MS m/z spectra obtained by reflectron negative or positive ion modes are reported in Figure 1. Detected compounds belonged to various classes including poly-unsaturated fatty acids (alpha/ gamma-linolenic acids, linoleic acid, oleic acid), saturated fatty acids (palmitic and stearic acids, miristic acid), high amounts of catechins and other anti-oxidants (caffeic acid; flavonoids apigenin, luteolin, and chrysoeriol; rosmarinic acid). These findings confirm the presence of a complex mixture of antioxidants as well as the presence of lipophilic components making antioxidants available into the cells.

\section{In vitro studies}

Cell viability (MTT test): Cell viability percentages are reported as mean \pm SD in Figure 2a. Either SDPE or HDPE used alone decreased cell viability as compared to control at any of the tested doses (50, 80, 160 ul per well). Conversely, $\mathrm{H}_{2} \mathrm{O}_{2}$ remarkably and significantly $(\mathrm{P}<0.001)$ decreased cell viability. SDPE and HDPE pretreatment were effective in protecting cells from $\mathrm{H}_{2} \mathrm{O}_{2}$ cytoxicity $(\mathrm{P}<0.001)$ reporting cell viability near to the value observed in control. This protective effect was observed also for co-treatment $(\mathrm{P}<0.05)$ and was only barely decreased in post-treatment (Figure 2b). Similar findings were obtained by analyzing TM cell morphology under the various experimental conditions tested (Figure 3a). Control cells maintained the stretched endothelial morphology; viability was good (as indicated by light absorbance making cells grey) as well as substrate adherence. In $\mathrm{H}_{2} \mathrm{O}_{2}$ treated cells morphology was changed into sphere-like and viability dramatically decreased (from $100 \%$ to $55 \%$ at MTT), substrate adherence was lost and light scattering made cells white and bright.

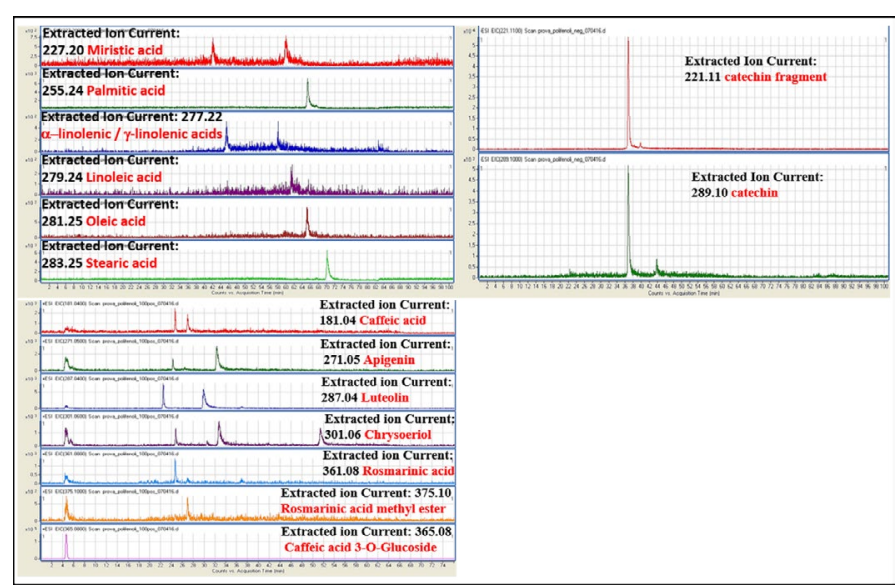

Figure 1. LC/MS analysis of $\mathrm{TTRAB} 囚$ (HDPE high-dose polyphenols and fatty acids extracts in hyaluronic acid $0.25 \%$ ), ranging from $100-2000 \mathrm{~m} / \mathrm{z}$, acquired in reflection negative (upper panels) or positive (lower panel) ion mode. The presence of selected fatty acids was evaluated by extracted ion current (EIC) based on their theoretical molecular weight $[\mathrm{M}-\mathrm{H}]^{-}$ 


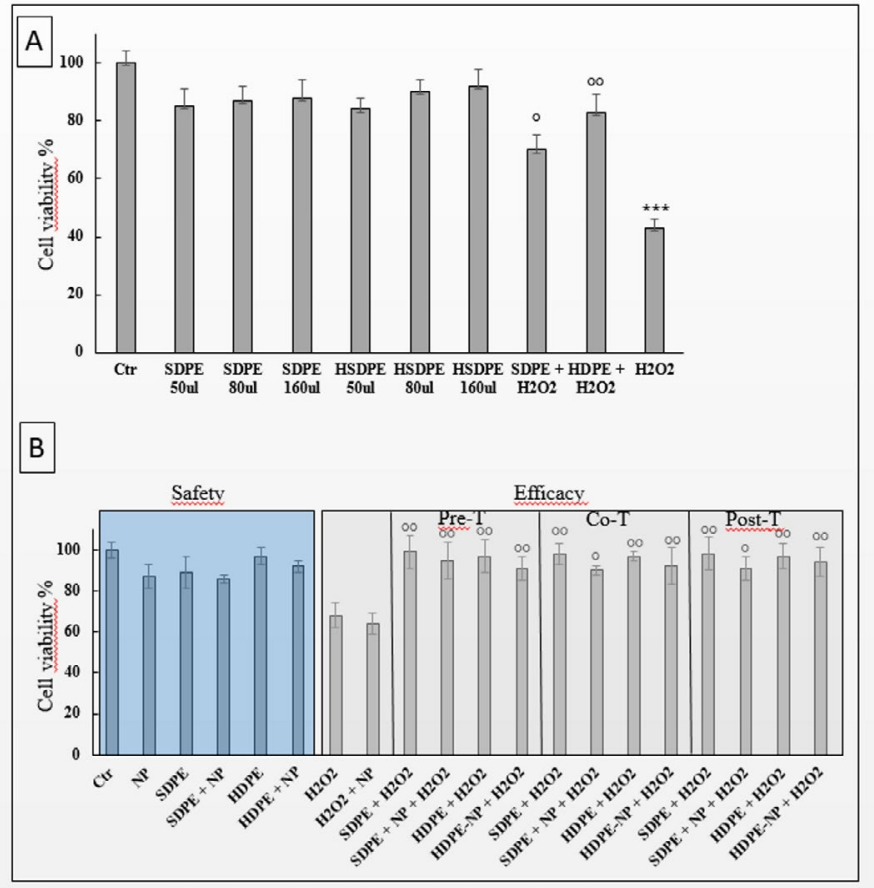

Figure 2. a) TM cell viability assay. Viability (mean \pm SD) of TM cells (MTT test) after treatments with either SDPE (standard dose) and HDPE (high-dose polyphenols and fatty acids extracts in hyaluronic acid $0.25 \%$ ), at increasing doses (50-160 ul per well). No doseresponse effect was observed. The dramatic decrease of cell viability induced by $\mathrm{H}_{2} \mathrm{O}_{2}$ was counteracted by SDPE and even more by HDPE. $* * * \mathrm{P}<0.001$ vs Control; ${ }^{\circ} \mathrm{P}<0.01$ and ${ }^{\circ} \mathrm{P}<0.05$ vs $\mathrm{H}_{2} \mathrm{O}_{2}$, b) Evaluation of eyedrop safety and efficacy. Safety was evaluated by comparing cell viability in untreated TM cells (control) versus cells treated with SPDE or HDPE not observing any significant change (left part). Efficacy was evaluated by comparing cell viability in TM cells undergoing oxidative damage $\left(\mathrm{H}_{2} \mathrm{O}_{2}\right)$ versus cells pre-, co- or post-treated with SDPE or HDPE observing significant decreases in cell mortality $\left({ }^{\circ} \mathrm{P}<0,05\right.$ and ${ }^{\circ \circ} \mathrm{P}<0,01$ versus $\left.\mathrm{H}_{2} \mathrm{O}_{2}\right)$. The effect of lipid nanoparticles (NP) used as SDPE and HDPE carrier did not modify either safety or efficacy of the eyedrop

Genotoxic damage (comet test): Examples of results obtained by Comet test are reported in Figure 3b. In control cells, DNA is not fragmented and does not migrate in the electrophoretic field, as indicated by rounded morphology of nuclei and lack of haloes and comet tails. Conversely, in $\mathrm{H}_{2} \mathrm{O}_{2}$ treated cells DNA is fully fragmented, nuclei dissolved and DNA spread into the electrophoretic field with massive formation of comets characterized by small nuclei and bulkystretched tails. These events are attenuated by HDPE both in pre- and co-treatment, with well-preserved nuclei; no bulky-stretched tail is detected. In co-treatment, nuclei are surrounded by haloes, a situation not detectable in pre-treatment. HDPE alone does not induce any DNA fragmentation in TM cells.

Microscope analysis of TM cell morphology: In HDPE pre-treated cells, only barely lost the stretched morphology, maintained substrate adherence as well as light absorbance, with cell viability of $96 \pm 8 \%$. In co-treated cells, morphology and light scattering were only partially altered, with cell viability of $97 \pm 12 \%$. In post-treated cells, HDPE attenuated $\mathrm{H}_{2} \mathrm{O}_{2}$-induced damage to a lesser extent than co- and pretreatment conditions, with a cell viability of $92 \pm 13 \%$. These findings indicate that HDPE is effective in protecting TM cells form oxidative damage and that pretreatment is more effective than co-treatment and post-treatment. No alteration in cell viability was detected when HDPE was used alone (viability $96 \pm 4 \%$ ).

The effect of charging HDPE onto NP was evaluated by MTT viability test in TM cells. NPs were tested at concentration ranging from 0.3 to $625 \mathrm{ug} / \mathrm{ml}$ (data not shown). Cell viability was decreased at concentration $>9.8 \mathrm{ug} / \mathrm{ml}$. Accordingly NP were used at $8 \mathrm{ug} / \mathrm{ml}$ concentration. No significant alteration in cells viability (viability $91 \pm$ $3 \%)$ or morphology was detected when HDPE-NP was used (Figure 4a, lower panel) as compared to HDPE alone (Fig. 4a, upper panel). The complexation of HDPE with NP slightly attenuated the protective effects exerted towards $\mathrm{H}_{2} \mathrm{O}_{2}$-induced damage (Figure 2, cell viability: pre-treatment $91 \pm 6 \%$, co-treatment $92 \pm 9 \%$, post-treatment $94 \pm 7 \%$ ).

This result provides evidence that NP do not modify HDPE safety .

\section{Ex vivo study: Evaluation of corneal distribution}

HDPE either alone (iTRAB ${ }^{ø}$ ) or complexed with NP (HDPE+NP, ERISnp *) were comparatively tested for their ability to penetrate corneal barrier in calf eye. Corneal distribution of HDPE labelled by

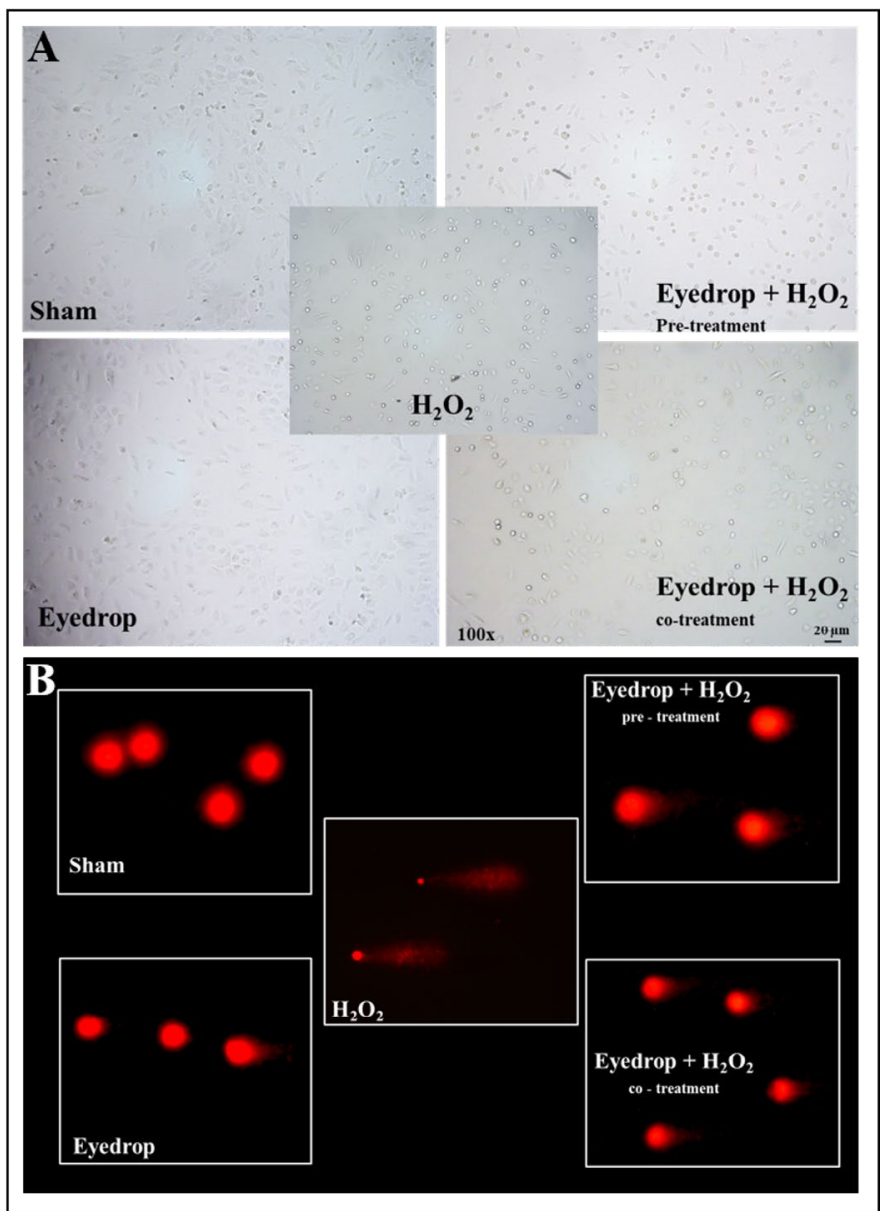

Figure 3. a) Microscopical appearance of $T M$ cell under the various experimental conditions tested. In both control and HDPE treatment (left panels) cells maintain the stretched endothelial morphology and good viability. In $\mathrm{H}_{2} \mathrm{O}_{2}$ treated cells (central panel) morphology becomes sphere-like and viability is dramatically decreased, as indicated by losing of adherence with substrate and light scattering.In HDPE pre-treated cells (upper right panel) substrate adherence is maintained and as well as cell viability. In HDPE cotreated cells (low right panel) both morphology as well as cell viability are only partially altered, b) Morphology of DNA fragmentation as evaluated by Comet test under the various experimental conditions tested. In both control and HDPE treated cells (left panels) DNA is not fragmented and does not migrate in the electrophoretic field, as indicated by rounded morphology of nuclei and lack of both haloes and comet tails. In $\mathrm{H}_{2} \mathrm{O}_{2}$ treatment (central panel) DNA is fully fragmented, nuclei dissolved, and DNA spread into the electrophoretic run, with massive formation of comets characterized by small nuclei and bulky-stretched tail. These events are attenuated by HDPE both in pre- and co-treatment (right panels) with well-preserved nuclei and no bulky-stretched tail. In co-treatment nuclei are surrounded by haloes a situation not detectable in pre-treatment 
Fluorescein was determined by slit lamp. An outline of the procedure used is reported in (Figure $4 \mathrm{~b}$ ). After 30 min since application, HDPE+NP does not cross the cornea but remain onto the anterior ocular surface. Conversely, HDPE penetrate in the corneal epithelium and, at least in part, diffuse to the posterior corneal surface and covering buffer. This figure was quantitatively determined by analyzing fluorescence recorded in the buffer covering posterior corneal surface in order to evaluate the amount of eye-drops penetrating through the cornea from ocular anterior surface to the anterior chamber (Figures $4 \mathrm{c}$ and $4 \mathrm{~d})$. The amount of fluorescence detected was $15.2 \pm 5.05$ for HDPE and $3.8 \pm 0.58$ for HDPE+NP (Fluorescence Units, mean \pm SD 4 experiments). Accordingly, HDPE was 4.0 -fold more effective than $\mathrm{HDPE}+\mathrm{NP}$ as far as concerns trans-corneal penetration.

\section{In vivo study}

No sign of inflammation was observed in conjunctiva or sclera of both eyes at any time interval either for HDPE or HDPE-NP in none of the 5 rabbits (Figure 5). The amount of eye drops remaining on ocular anterior surface was comparatively evaluated between HDPE (upper row) and HDPE+NP (lower row) by analyzing blue light fluorescence. Eyedrops amount was similar at 5, 10, min; a decrease for HDPE only was observed since $20 \mathrm{~min}$. After $20 \mathrm{~min}$, the remaining amount of $\mathrm{HDPE}+\mathrm{NP}$ was consistently higher than those of HDPE at any time tested. After $4 \mathrm{~h}$ only small traces of HDPE were detectable in conjunctival bag, indicating the full clearance of the eyedrop; conversely HDPE+NP was still well detectable onto corneal surface. After $16 \mathrm{~h}$ HDPE was completely removed from the eye while HDPE+NP was still abundantly detectable onto corneal surface (Figure 5). This finding indicate that HDPE+NP remain onto corneal surface much longer than HDPE.

\section{Discussion}

Presented results provide evidence that eye drops containing a mixture from Perilla frutescens extracts at certain concentrations of flavonoids, catechins and fatty acids at the tested doses are devoid of direct toxic effect on TM cells while being able to protect them from oxidative stress. A similar safety was observed both for SDPE and HDPE. This finding is of relevance due to the high susceptibility of TM cells to oxidative damage [6] and to the pathogenic role of oxidative stress in glaucoma [31]. TM shows a striking morphological decay both with age and during the course of glaucoma. Indeed, the glaucomatous TM is prematurely aged. Therefore, protecting these cells by polyphenols means better TM efficacy and hence a smaller number of proapoptotic signals reaching the optic nerve head.

HDPE contains 2,3-dihydroxy-4,6-dimethoxychalcone that is an activator of the nuclear factor erythroid 2-related factor 2 (Nrf2)antioxidant response element (ARE) pathway, which is a cellular defense system against oxidative stress, controlling hundreds of detoxifying and antioxidant, and preventing dopaminergic neuron death from oxidative stress by upregulation of glial expression of heme oxygenase-1 (HO-1), one of the ARE-driven antioxidant enzymes [32]. The Nrf2 gene is an important factor in protecting RGCs from axonal damage induced RGCs death because of oxidative stress conditions and depletion of Nrf2 aggravates RGC death induced by optic nerve injury [33]. Indeed, the Nrf2 pathway appears to be a promising target for glaucoma neuroprotection [34,35].

Mitochondrial function plays a fundamental role in glaucoma pathogenesis, because they represent the greatest source of free radicals in the TM, especially as related to POAG and pseudo-exfoliative

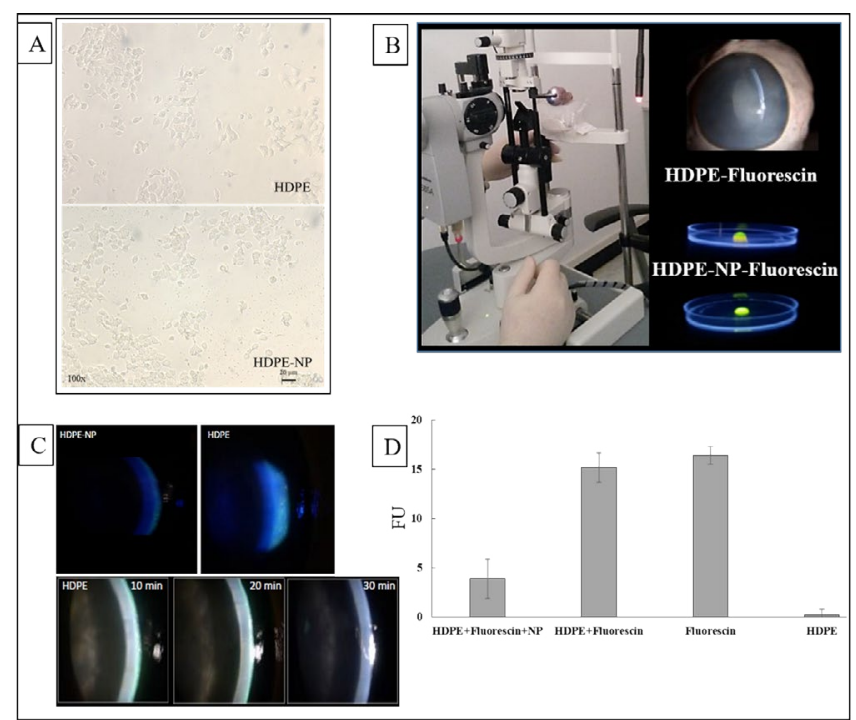

Figure 4. a) TM cell viability assay. Viability (mean \pm SD) of TM cells (MTT test) after treatments with either SDPE and iTRAB ${ }^{\circ}$ HDPE high-dose polyphenols and fatty acids extracts in hyaluronic acid $0.25 \%$, at increasing doses (50-160 ul per well). No doseresponse effect was observed, b) Microscope analysis of TM cell morphology. Microscope analysis of TM cell morphology treated with HDPE (upper panel) or HDPE-NP (lower panel). In the lower panel NP have been aggregated by short-time DMSO treatment to make $\mathrm{NP}$ aggregates visible (dark dots). Cell morphology and viability is not altered by NP whose use does not modify HDPE safety and efficacy, c) Outline of the procedure used to evaluate trans-corneal permeability in the ex-vivo experiment. HDPE or HDPE-NP were labelled by fluorescein and dropped onto the corneal anterior surface of a calf eye collected from cadaver. After $30 \mathrm{~min}$ trans corneal distribution was evaluated by slit lamp under blue light irradiation. Corneal absorption of HDPE-NP was very weak while HDPE was consistently detectable into corneal layers and beyond the inner corneal surface (upper panels), d) The time-course of HDPE corneal absorption was evaluated at 10, 20,30 min observing a time dependent corneal absorption (lower panels). The amount of eyedrop crossing through cornea was evaluated by quantitative fluorescence (right histogram). No fluorescence was detected in blank and NP samples, high quantity of fluorescence was observed for HDPEfluorescein and only small quantity for HDPE-NP
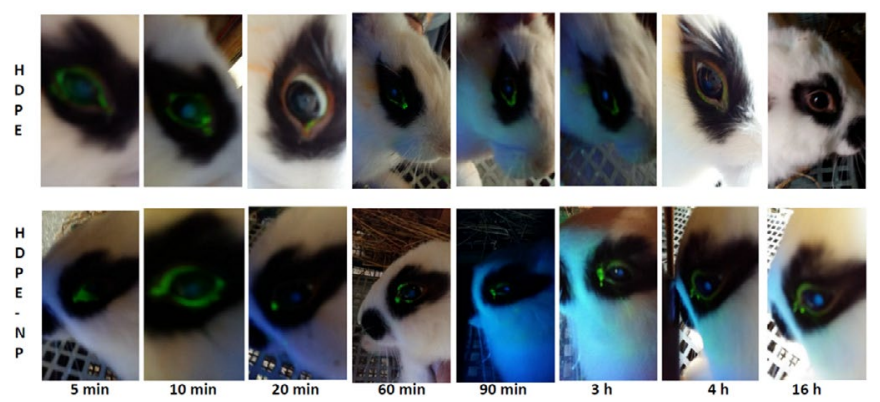

Figure 5. Comparative evaluation of HDPE or HDPE-NP time persistence onto corneal outer surface in rabbit. Comparative evaluation of HDPE (right eye, upper row) or HDPENP (left eye, lower row) time persistence onto corneal outer surface in rabbit. The amount of fluorescein-labelled eye drops was determined by fluorescence under blue light. Eyedrop amounts were similar for HDPE and HDPE-NP at 5, 10, 20, min with a clear decrease at $20 \mathrm{~min}$ for HDPE only. The amount of HDPE-NP was higher than HDPE at 1, 2, 3 $\mathrm{h}$. After $4 \mathrm{~h}$ only small traces of HDPE were detectable in conjunctival bag due to its corneal absorption, while HDPE-NP was still well detectable onto corneal surface. After $16 \mathrm{~h}$ HDPE was completely removed from the eye while HDPE-NP was still abundantly detectable onto corneal surface

glaucoma [36]. None adverse effect on TM cells was observed, evaluating mitochondrial function by MTT, as induced either by SDPE or HDPE. No increase in DNA fragmentation was observed as induced by either SDPE or HDPE at comet test, an extremely sensitive assay. The safety polyphenolic eyedrops was confirmed by in vivo experiments where no sign of inflammation or other adverse effect (e.g., increase of blinking frequency or tearing) was detected in rabbit. HDPE is a rich 
source of omega-3 polyunsaturated fatty acids (PUFAs) that have antiinflammatory, antithrombotic, lipid lowering, vasodilatory, and neuro protective capacities [37,38]. The role of omega 3 fatty acid in down regulating inflammation is an important paradigm for understanding the pathogenesis of chronic inflammatory diseases including macular degeneration, glaucoma and dry eye [39].

The high susceptibility of TM cells to oxidative damage together with the toxic action of preservatives contained in anti-glaucoma drugs are chronically combined and causing cell damage as it occurs in the anterior surface and TM [40]. These adverse effects are prevented when TM cells were treated with either SDPE or, even more, HDPE. Indeed, HDPE was more protective than SDPE in co- and post-treatment, while no difference was observed for pre-treatment.

Different protective mechanisms are triggered according to the experimental condition tested. In co-treatment, the direct neutralization of reactive oxygen species by polyphenolic components both in extra-and intra-cellular compartment prevails. In pretreatment polyphenolic compounds activate anti-oxidant intracellular defenses, mainly including phase II protective pathways, that are ready to react when oxidative stress occurs. The protective effect observed in post-treatment is somewhat more surprising being not amenable to none of these two mechanisms. This situation could be explained by the activation by eyedrop components of cellular mechanisms able to repair $\mathrm{H}_{2} \mathrm{O}_{2}$-induced damage, this figure including DNA and protein repair. Indeed, several components of tested eyedrop are able to trigger these mechanisms as demonstrated for polyphenols and repair of DNA damage induced by UV light [41]. Similar results have been obtained for polyphenols and protein repair evaluating lipofuscin formation in an experimental model of macular degeneration triggered by blue light [42].

Herein reported results are in line with experimental evidences dealing the use of polyphenols as anti-oxidant protecting from lipid peroxidation in experimental tests and humans [43]. This direct anti-oxidant activity is further enhanced by the anti-inflammatory properties of components of Perilla extracts as luteolin, inflammation being a main endogenous source of oxidative damage.

The use of NPs did not modify neither safety or efficacy of HDPE as indicated by MTT and microscope analysis on TM cells. This finding indicates that HDPE is not irreversibly sequestrated into NPs but is reversibly adsorbed onto the outer lipophilic membrane of NP that functions as carriers for the eyedrop. When NPs target cell membrane the eyedrop is released into the intracellular environment, a mechanism well established for this type of NPs when used as carriers for polyphenolic compounds such as resveratrol [30]. Under the experimental situation tested, NP do not increase HDPE efficacy that is already at highest level when HDPE was used alone. This finding indicates that HDPE is per se highly bioavailable due to its intrinsic high lipophilicity. However, the in vivo experiment demonstrates that NP modifies the pharmacokinetic properties of HDPE. HDPE-NP was unable to permeate corneal barrier in ex-vivo experiment at variance with HDPE. This event is attributable to the fact that lipid NPs further increase lipophilicity and viscosity of HDPE thus rendering eye-drop more persistent on the outer corneal surface despite the existence of effective drainage mechanisms. Indeed, HDPE was removed from cornea already after $20 \mathrm{~min}$ that is already a relatively long persistence time considering that hydrophilic eye-drops are removed by blinking and tearing after only few minutes since application [44].

On the whole obtained results indicates that HDPE is able to permeate cornea thus increasing the amount of protective anti-oxidant in ocular anterior chamber. These mechanisms are useful to protect fragile TM cells form oxidative stress, a driving force of POAG, where the aqueous humor from levels of anti-oxidant enzymes are significantly lowered in glaucoma [45].

An alternative strategy to increase eyedrops bioavailability in the anterior chamber is the inclusion in the eye drops of aggressive component such as benzalkonium chloride that, transiently damaging cornea, increase the ability of eye-drop to cross cornea. However, benzalkonium chloride and other anti-bacterial agents used at this purpose are highly toxic for TM [10] and induce inflammation in conjunctiva [46]. Conversely, HDPE increase polyphenols bioavailability for the ocular anterior chamber in a safe manner due to their unsolubility in the hydrophilic tear film and their ability to adhere onto corneal outer surface and to cross corneal barrier. The long persistence and high bioavailability of eye-drop increase pharmacodynamic properties thus decreasing the number and frequency of application required to obtain the therapeutic and preventive effects.

\section{Conclusions}

Our results provide evidence that eyedrop containing hyaluronic acid with high molecular weight and a complex mixture of polyphenols are: (1) safe especially for cells sensitive to oxidative stress such as TM cells; (2) able to protect TM cells from oxidative damage, a driving mechanism of glaucoma pathogenesis; (3) more potent than hydrophilic eyedrops; (4) remain for a long time onto outer corneal surface after application especially when complexed with NP; (5) when used uncomplexed with NP, are able to cross corneal barrier thus delivering anti-oxidant into ocular anterior chamber and aqueous humor, whose composition is altered in glaucoma. Accordingly, lipohilic eyedrops containing polyphenols extracts appear to be of interest for the prevention of diseases targeting ocular anterior surface and anterior chamber.

\section{Acknowledgments}

We thank Mr Luigi Gallato for his kind collaboration in in vivo experiments. This study was kindly supported by Eyepharma SpA, Genoa, Italy.

\section{Declaration of interest}

None.

\section{References}

1. Sacca SC, Gandolfi S, Bagnis A, Manni G, Damonte G, et al. (2016a) From DNA damage to functional changes of the trabecular meshwork in aging and glaucoma. Ageing Res Rev 29: 26-41. [Crossref]

2. Sacca SC, Gandolfi S, Bagnis A, Manni G, Damonte G, et al. (2016) The outflow pathway: A tissue with morphological and functional unity. J Cell Physiol 231: 18761893. [Crossref]

3. Izzotti A, Saccà SC, Cartiglia C, De Flora S (2003) Oxidative deoxyribonucleic acid damage in the eyes of glaucoma patients. Am J Med 114: 638-646. [Crossref]

4. Izzotti A, Longobardi MG, Cartiglia C, Sacca SC (2010) Mitochondrial damage in the trabecular meshwork occurs in primary open-angle glaucoma and in pseudoexfoliative glaucoma. Plos One 6: e14567. [Crossref]

5. Pulliero A, Seydel A, Camoirano A, Sacca SC, Sandri M, et al. (2014) Oxidative damage and autophagy in the human trabecular meshwork as related with ageing. PLoS One 9: e98106. [Crossref]

6. Izzotti A, Sacca SC, Longobardi M, Cartiglia C (2009) Sensitivity of ocular anterior chamber tissues to oxidative damage and its relevance to the pathogenesis of glaucoma. Invest Ophthalmol Vis Sci 50: 5251-5258. [Crossref] 
7. Sacca SC, Pascotto A, Camicione P, Capris P, Izzotti A (2005) Oxidative DNA damage in the human trabecular meshwork. Clinical correlations in patients with primary open angle glaucoma. Arch Ophthalmol 123: 458-463. [Crossref]

8. Sacca SC, Izzotti A (2008) Oxidative stress and glaucoma: Injury in the anterior segment of the eye. Prog Brain Res 173: 385-407. [Crossref]

9. Sacca SC1, Izzotti A (2014) Focus on molecular events in the anterior chamber leading to glaucoma. Cell Mol Life Sci 71: 2197-2218. [Crossref]

10. Izzotti A, La Maestra S, Micale RT, Longobardi MG, Sacca SC (2015) Genomic and post-genomic effects of anti-glaucoma drugs preservatives in trabecular meshwork. Mutat Res 772: 1-9. [Crossref]

11. Tezel G, Yang X (2004) Caspase-independent component of retinal ganglion cell death, In vitro. Invest Ophthalmol Vis Sci 11: 4049-4059. [Crossref]

12. Crawford ML, Harwerth RS, Smith EL 3rd, Mills S, Ewing B (2001) Experimenta glaucoma in primates: changes in cytochrome oxidase blobs in V1 cortex. Invest Ophthalmol Vis Sci 42: 358-364. [Crossref]

13. Chua J, Vania M, Cheung CM, Ang M, Chee SP, et al. (2012) Expression profile of inflammatory cytokines in aqueous from glaucomatous eyes. Mol Vis 18: 431-438. [Crossref]

14. Sacca SC, Centofanti M, Izzotti A (2012) New proteins as vascular biomarkers in primary open angle glaucomatous aqueous humor. Invest Ophthalmol Vis Sci 53: 42424253. [Crossref]

15. Ren H, Magulike N, Ghebremeskel K, Crawford M (2006) Primary open-angle glaucoma patients have reduced levels of blood docosahexaenoic and eicosapentaenoic acids. Prostaglandins Leukot Essent Fatty Acids 74: 157-163. [Crossref]

16. Pérez de Arcelus M, Toledo E, Martinez-Gonzalez MA, Sayon-Orea C, Gea A, et al. (2014) Omega 3:6 ratio intake and incidence of glaucoma: the SUN cohort. Clin Nutr 33: 1041-1045. [Crossref]

17. Garcia-Medina JJ, Garcia-Medina M, Garrido-Fernandez P, Galvan-Espinosa J, GarciaMaturana C, et al. (2015) A two-year follow-up of oral antioxidant supplementation in primary open-angle glaucoma: an open-label, randomized, controlled trial. Acta Ophthalmol 93: 546-554. [Crossref]

18. Quaranta L, Bettelli S, Uva MG, Semeraro F, Turano R, et al. (2003) Effect of Ginkgo Biloba extract on pre-existing visual field damage in normal tension glaucoma Ophthalmology 110: 359-362. [Crossref]

19. Szumny D, Sozanski T, Kucharska AZ, Dziewiszek W, Piórecki N, et al. (2015) Application of cornelian cherry iridoid-polyphenolic fraction and loganic acid to reduce intraocular pressure. Evid Based Complement Alternat Med.

20. Falsini B, Marangoni D, Salgarello T, Stifano G, Montrone L, et al. (2009) Effect of epigallocatechin-gallate on inner retinal function in ocular hypertension and glaucoma: A short-term study by pattern electroretinogram. Graefes Arch Clin Exp Ophthalmol 247: 1223-1233. [Crossref]

21. Bola C, Bartlett H, Eperjesi F (2014) Resveratrol and the eye: Activity and molecular mechanisms. Graefes Arch Clin Exp Ophthalmol 252: 699-713. [Crossref]

22. Curin Y, Andriantsitohaina R. (2005) Polyphenols as potential therapeutical agents against cardiovascular diseases. Pharmacol Rep 57s: 97-107. [Crossref]

23. Corder R, Douthwaite JA, Lees DM, Khan NQ, Viseu Dos Santos AC, et al. (2001) Endothelin-1 synthesis reduced by red wine. Nature 414: 863-864. [Crossref]

24. Beckman $\mathrm{CH}$ (2000) Phenolic-storing cells: Keys to programmed cell death and periderm formation in wilt disease resistance and in general defence responses in plants? Physiol Mol Plant Pathol 57: 101-110.

25. Graf BA, Milbury PE, Blumberg JB (2005) Flavonols, flavonones, flavanones and human health: Epidemological evidence. J Med Food 8: 281-290. [Crossref]

26. Arts IC, Hollman PC (2005) Polyphenols and disease risk in epidemiologic studies. Am J Clin Nutr 81: 317S-325S. [Crossref]
27. Manach C, Scalbert A, Morand C, Rémésy C, Jiménez L (2004) Polyphenols: Food sources and bioavailability. Am J Clin Nutr 79: 727-747. [Crossref]

28. Coleman AL, Stone KL, Kodjebacheva G, Yu F, Pedula KL, et al. (2008) Glaucoma risk and the consumption of fruits and vegetables among older women in the study of osteoporotic fractures. Am J Ophthalmol 145: 1081-1089. [Crossref]

29. Giaconi JA, Yu F, Stone KL, Pedula KL, Ensrud KE, et al. (2012) The association of consumption of fruits/vegetables with decreased risk of glaucoma among older African-American women in the study of osteoporotic fractures. Am J Ophthalmol 154: 635-644. [Crossref]

30. Pulliero A, Wu Y, Fenoglio D, Parodi A, Romani M, et al. (2015) Nanoparticles increase the efficacy of cancer chemopreventive agents in cells exposed to cigarette smoke condensate. Carcinogenesis 36: 368-377. [Crossref]

31. Izzotti A, Bagnis A, Saccà SC (2006) The role of oxidative stress in glaucoma. Mutat Res 612: 105-114. [Crossref]

32. Sitte N, Merker K, Von Zglinicki T, Grune T (2000) Protein oxidation and degradation during proliferative senescence of human MRC-5 fibroblasts. Free Radic Biol Med 28: 701-708. [Crossref]

33. Masaki Y, IzumiY, Matsumura A, Akaike A, Kume T (2017) Protective effect of Nrf2ARE activator isolated from green perilla leaves on dopaminergic neuronal loss in a Parkinson's disease model. Eur J Pharmacol 798: 26-34. [Crossref]

34. Himori N, Yamamoto K, Maruyama K, Ryu M, Taguchi K, et al. (2013) Critical role of $\mathrm{Nrf} 2$ in oxidative stress-induced retinal ganglion cell death. $J$ Neurochem 127: 669680. [Crossref]

35. Xu Z, Cho H, Hartsock MJ, Mitchell KL, Gong J, et al. (2015) Neuroprotective role of $\mathrm{Nrf} 2$ for retinal ganglion cells in ischemia-reperfusion. J Neurochem 133: 233-241. [Crossref]

36. Batliwala S, Xavier C, Liu Y, Wu H, Pang IH (2017) Involvement of Nrf2 in ocular diseases. Oxid Med Cell Longev 2017: 1703810. [Crossref]

37. Asif M (2011) Health effects of omega-3,6,9 fatty acids: Perilla Frutescens is a good example of plant oils. Orient Pharm Exp Med 11: 51-59. [Crossref]

38. Den Ruijter HM, Berecki G, Opthof T, Verkerk AO, Zock PL, et al. (2007) Proand antiarrhythmic properties of a diet rich in fish oil. Cardiovasc Res 73: 316-325. [Crossref]

39. Wall R, Ross RP, Fitzgerald GF, Stanton C (2010) Fatty acids from fish: the antiinflammatory potential of long-chain omega-3 fatty acids. Nutr Rev 68: 280-289. [Crossref]

40. Walter SD, Gronert K, McClellan AL, Levitt RC, Sarantopoulos KD, et al. (2016) $\omega-3$ tear film lipids correlate with clinical measures of dry eye. Invest Ophthalmol Vis Sci 57: 2472-2478. [Crossref]

41. Servat JJ, Bernardino CR (2011) Effects of common topical antiglaucoma medications on the ocular surface, eyelids and periorbital tissue. Drugs Aging 28: 267-282. [Crossref]

42. Katiyar SK, Vaid M, van Steeg H, Meeran SM (2010) Green tea polyphenols prevent UV-induced immunosuppression by rapid repair of DNA damage and enhancement of nucleotide excision repair genes. Cancer Prev Res 3: 179-189. [Crossref]

43. Zhao Z, Sun T, Jiang Y, Wu L, Cai X, et al. (2014) Photooxidative damage in retina pigment epithelial cells via GRP78 and the protective role of grape skin polyphenols. Food Chem Toxicol 74: 216-224. [Crossref]

44. Bagnis A, Izzotti A, Centofanti M, Saccà SC (2012) Aqueous humor oxidative stress proteomic levels in primary open angle glaucoma. Exp Eye Res 103: 55-62. [Crossref]

45. Schirrmacher G, Skurk T, Hauner H, Grassmann J (2010) Effect of Spinacia oleraceae L. and Perilla frutescens L. on Antioxidants and lipid peroxidation in an intervention study in healthy individuals. Plant Foods Hum Nutr 65: 71-76. [Crossref]

46. Urtti A (2006) Challenges and obstacles of ocular pharmacokinetics and drug delivery. Adv Drug Deliv Rev 58: 1131-1135. [Crossref]

Copyright: (C2019 Sacca SC. This is an open-access article distributed under the terms of the Creative Commons Attribution License, which permits unrestricted use, distribution, and reproduction in any medium, provided the original author and source are credited. 